\title{
The Occurrence of Aging-Dependent Reticulon 3 Immunoreactive Dystrophic Neurites Decreases Cognitive Function
}

\author{
Qi Shi, ${ }^{\star}$ Xiangyou Hu, ${ }^{\star}$ Marguerite Prior, and Riqiang Yan \\ Department of Neurosciences, Lerner Research Institute, Cleveland Clinic, Cleveland, Ohio 44195
}

Reticulon 3 (RTN3) has been shown to mark a distinct and abundant population of dystrophic neurites named RTN3 immunoreactive dystrophic neurites (RIDNs) in patients' brains of Alzheimer disease (AD). Transgenic mice expressing RTN3 (Tg-RTN3) also spontaneously develop RIDNs. To determine whether RIDNs formed in Tg-RTN3 mice would ever naturally occur in the nontransgenic mouse brain, we targeted our examination to elderly mouse brains on the basis that $\mathrm{AD}$ is an age-dependent neurodegenerative disease where the decline in cognitive function becomes progressively increased during the course of the disease. Here, we demonstrate that the distribution of RIDNs is abundant, rather than sporadic, in elderly but not young mouse brains. RIDNs in the elderly brain have two distinct populations: abundantly dispersed RIDNs that can only be marked by RTN3, and less abundantly clustered RIDNs that can be marked by multiple proteins including RTN3, ubiquitin, and phosphorylated neurofilament. The abundance of RIDNs in Tg-RTN3 mice at the age of 3 months resembles that of 24-month-old wild type mice, suggesting that this animal model mimics and accelerates the natural occurrence of RIDNs. Importantly, we demonstrate that preformed RIDNs appear to reduce dendritic spine density and synaptic function. Further analysis from mechanistic studies suggests that elevated levels of RTN3 lead to an imbalance in the axonal transport of RTN3, which results in the accumulation of RTN3 in swollen neurites. Collectively, these results suggest that blocking the formation of RIDNs may be a promising strategy to impede cognitive decline in the elderly and in AD patients.

\section{Introduction}

Dystrophic neurites, defined as aberrant neuritic sproutings, swollen dendrites, and/or swollen axons, are often found in the brains of patients suffering from neurodegenerative diseases. For example, dystrophic neurites in the brains of patients with Alzheimer's disease (AD) are found mainly in the area surrounding neuritic amyloid plaques (Dickson et al., 1988; Lenders et al., 1989; Onorato et al., 1989; Hu et al., 2007). Morphological characterizations of dystrophic neurites in AD brains have been extensively described, and many proteins, including ubiquitin (Kowall and Kosik, 1987; Perry et al., 1987; Onorato et al., 1989) and GAP-43 (Masliah et al., 1992), can mark dystrophic neurites. In Parkinson's and Lewy body diseases, dystrophic nigral neurites can be marked by an $\alpha$-synuclein antibody (Irizarry et al., 1998). Dystrophic neurites have also been observed around prionic protein-derived amyloid plaques of transmissible cerebral amyloidoses of kuru, Creutzfeldt-Jakob disease, Gerstmann-

Received Dec. 11, 2008; revised March 17, 2009; accepted March 19, 2009.

This work was partially supported by National Institutes of Health Grant AG025493 to R.Y., and awards from Ralph Wilson Foundation and Alzheimer's Association to R.Y. A postdoctoral fellowship from American Health Assistance Foundation is awarded to M.P. We wish to thank anonymous reviewers for their constructive comments, especially the suggestion for the experiment of sciatic nerve ligation, and Dr. Grahame Kidd for assistance with spine images.

${ }^{*}$ Q.S. and X.H. contributed equally to this work.

Correspondence should be addressed to Dr. Riqiang Yan, Department of Neurosciences, Lerner Research Institute, Cleveland Clinic, 9500 Euclid Avenue, Cleveland, OH 44195. E-mail: yanr@ccf.org.

DOI:10.1523/JNEUROSCI.5887-08.2009

Copyright $\odot 2009$ Society for Neuroscience $\quad 0270-6474 / 09 / 295108-08 \$ 15.00 / 0$
Sträussler-Scheinker disease, and scrapie (Migheli et al., 1991; Liberski, 1995). Contrary to the extensive morphological characterizations of dystrophic neurites, the molecular mechanism underlying their formation is less understood. Axonopathy or neuronal injury is often believed to cause neuritic dystrophy. Factors such as the impairment of axonal transport (Stokin et al., 2005), impaired membrane fragility (Praprotnik et al., 1996), or cytoskeletal alterations (Dickson et al., 1999) that occur in neurodegenerative diseases are suggested to facilitate the formation of dystrophic neurites.

Our research interests in the formation of dystrophic neurites are based on our recent finding that reticulon 3 (RTN3), one of the four members of the Nogo/reticulon protein (RTN) family (Oertle and Schwab, 2003; Yan et al., 2006), is markedly accumulated within dystrophic neurites in the brains of $\mathrm{AD}$ patients and amyloid precursor protein (APP) transgenic mice ( $\mathrm{Hu}$ et al., 2007). Morphological characterizations indicate that RTN3 marks a distinct population of dystrophic neurites, and we have named these as RTN3 immunoreactive dystrophic neurites (RIDNs) for two reasons. First, no other protein has yet been identified to completely coexist with RTN3 in RIDNs. Biochemical and ultrastructural studies suggest that RIDNs are enriched with RTN3 aggregates (Hu et al., 2007). Second, RIDNs are significantly more abundant than dystrophic neurites labeled by many reported markers in AD brains. More importantly, transgenic mice overexpressing only RTN3 (Tg-RTN3), but not the aforementioned proteins, spontaneously produce RIDNs, fur- 
ther indicating that RIDNs can be formed without association with other neurodegenerative hallmarks.

To determine whether RIDNs formed in Tg-RTN3 mice would ever naturally occur in the nontransgenic mouse brain, we screened for the presence of RIDNs in elderly brains. Strikingly, we found that RIDNs were indeed present in the elderly mouse brain hippocampal CA1 region, but not in the CA1 of young mouse brains. We also showed that preformed RIDNs cause a loss of dendritic spine density. Our results not only reveal the natural occurrence of RIDNs in the elderly brain, but also suggest that the presence of RIDNs in the elderly brain potentially contributes to the reduced cognitive function associated with aging. Hence, our findings suggest that reducing the formation of RIDNs is a novel therapeutic strategy with the aim of reducing the decline in cognitive function in the elderly population and in $\mathrm{AD}$ patients.

\section{Materials and Methods}

Immunofluorescent confocal microscopy. Mouse brain tissues were first fixed with $4 \%$ paraformaldehyde and were then cut on a freezing microtome (Microm). Confocal staining of the fixed brain sections was performed according to published procedures (Hu et al., 2007). RTN3 immunoreactivity was detected with polyclonal R458 (1:1000) (He et al., 2004 ). Antibodies against $\beta$-amyloid peptide (6E10 and 4G8), APP, and ubiquitin were purchased from Sigma; actin from Santa Cruz Biotechnology; AT8 for phosphorylated tau from Innogenetics-Biotechnology for Health; and SMI 31 and SMI 32 from Sternberger Monoclonal. Alexa488 and Alexa-568 labeled secondary antibodies (1:200) were purchased from Invitrogen.

Western blot analysis of brain samples. Mouse brain samples were prepared for Western blots as follows. First, fresh samples were homogenized on ice in a solution containing $50 \mathrm{~mm}$ Tris- $\mathrm{HCl}(\mathrm{pH}=7.5), 150 \mathrm{~mm}$ $\mathrm{NaCl}, 0.5 \% \mathrm{NP}-40$, and $1 \times$ protease inhibitor cocktail (Roche Biosciences). In the case of complete dissociation of RTN3 aggregates, $1 \%$ CHAPS detergent was used in place of $0.5 \%$ NP-40. The homogenates were incubated on a rotator for $30 \mathrm{~min}$ at $4^{\circ} \mathrm{C}$. After removal of tissue debris by centrifugation, the protein concentrations of the supernatants were determined with the Bradford protein assay kit (Bio-Rad Laboratories). Lysates were prepared to $30 \mu \mathrm{g}$ of protein, and were then separated on a $4-12 \%$ Bis-Tris NuPage gel (Invitrogen), followed by Western blotting with R458 or the control antibodies specific to calnexin. Immunoblots were visualized using enhanced chemiluminescence (Thermo Scientific).

Sciatic nerve ligation and Western blot. After sodium pentobarbitalinduced anesthesia according to the standard procedure, the skin on the lateral surface of the right thigh was incised, and a section was made directly through the biceps femoris muscle. The sciatic nerve was exposed and tightly ligated using 6.0 silk sutures (Ethicon). Six or 20 h later, segments proximal and distal to the ligature were collected and frozen on dry ice. The contralateral nerve was kept intact as a control. The nerve segments were homogenized in modified RIPA buffer $[50 \mathrm{~mm}$ Tris- $\mathrm{HCl}$, pH 7.4, 1\% NP-40, 0.25\% Na-deoxycholate, 150 mu NaCl, 1 mm EDTA, $1 \mathrm{~mm} \mathrm{Na}_{3} \mathrm{VO}_{4}, 1 \mathrm{~mm} \mathrm{NaF}$, and protease inhibitor cocktail (Roche)] and centrifuged at 13,000 $\mathrm{rpm}$ for $1 \mathrm{~h}$. The protein concentration of the supernatant was determined using Bio-Rad protein assay. Equal amounts of protein $(40 \mu \mathrm{g})$ were resolved on a NuPAGE Bis-Tris Gel (Invitrogen) and transferred onto nitrocellulose membranes (Invitrogen). Subsequently, blots were incubated with primary antibodies (RTN3, 1:1000; P75, 1:1000; APP, 1:1000; synapsin-I, 1:1000; $\beta$-actin, $1: 10,000)$ overnight at $4^{\circ} \mathrm{C}$. After extensive wash, the blots were reacted with HRP-conjugated secondary antibodies and visualized using enhanced chemiluminescence (Thermo Scientific). The ratios of RTN3 by calnexin were calculated from three independent experiments and were plotted.

Golgi staining, spine density imaging, and calculation. Golgi staining was performed according to the manufacturer's instructions in the FD Rapid GolgiStain Kit (FD NeuroTechnologies, Consulting \& Services). Mice were killed, followed by immersion of a fresh mouse brain hemi- sphere in impregnation solution (a mixture of solution A and solution B in equal amounts) for 2 weeks. After this period, brain samples were transferred to solution $\mathrm{C}$ as provided in the kit and stored at room temperature away from light for $2 \mathrm{~d}$. For sectioning, the impregnated hemispheres were first embedded in isopentane on dry ice, and the embedded brains were then sectioned at a thickness of $50-60 \mu \mathrm{m}$ on a freezing microtome $\left(-22^{\circ} \mathrm{C}\right)$. Sectioned specimens were mounted onto microscope slides that were precoated with $5 \%$ gelatin. Sections were rinsed in water twice, for $2 \mathrm{~min}$ each, and then incubated in a mixture of solution $\mathrm{D}$, solution E, and water (50, 50 and $100 \mathrm{ml}$, respectively) for $10 \mathrm{~min}$. Sections were then rinsed in distilled water twice for $4 \mathrm{~min}$ each, and were also counterstained with cresyl violet. Gradual dehydration was performed using 50, 75, 85, 95, and 100\% ethanol (twice), for 4 min each. Sections were then cleared in $100 \%$ xylene three times, for 4 min each. Finally, specimens were sealed with coverslips in Permount mounting medium and dried at room temperature.

The specimens were examined using the Zeiss Axiophot fluorescence microscope system. Dendritic branches with spines were imaged through a $\times 63$ lens (numerical aperture, 1.4) using a MagnaFIRE digital camera. A through-focus series of color images of each dendritic branch were captured. In ImageJ stacks, each set of the color images were sharpened and then $z$-projected to produce a final image. The microscopy and computer software were calibrated to measure the length of dendritic branches in micrometers. Dendritic spines were randomly selected and counted in a blinded manner.

Quantification of RIDNs and statistic analysis. To quantify RIDNs, we performed confocal staining of brain sections of $10 \mu \mathrm{m}$ thickness with primary rabbit anti-RTN3 antibody and secondary anti-rabbit IgG antibody conjugated with Alexa 488 fluorophore. A stack of 10 images in $\mathrm{Z}$-series that covers the entire section was taken, and these 10 images were then combined into one image for quantification. ImageJ software was used to quantify the area of RIDNs in the hippocampal CAl region. Typically, the signals of RIDNs are bright, whereas normal staining of cells by RTN3 antibody in hippocampus is relatively dim. Only RIDNs were selected for quantification, whereas staining of neurons by RTN3 was excluded. For each mouse, one in every eight continuous brain sections was stained and quantified. Percentage of RIDNs area was calculated by dividing the total RIDNs area by the total examined hippocampal area in each mouse. $t$ tests were used to compare two groups of data. One-way ANOVA was performed to compare three groups or more of data. A $p$ value of $<0.05$ was considered to be statistically significant.

\section{Results}

\section{The occurrence of RIDNs in the elderly mouse brain}

Although the occurrence of dystrophic neurites is often described as a secondary event to the so-called pathological hallmarks of neurodegenerative diseases, i.e., amyloid plaques, their occurrence marked by antibodies against ubiquitin, APP, or neurofilament in normal elderly brains appears to be relatively rare (Dickson et al., 1990; Migheli et al., 1992; Wang and Munoz, 1995; Vickers et al., 1996). Based on the findings that RIDNs represent an abundant form of dystrophic neurites in $\mathrm{AD}$ brains and that Tg-RTN3 mice can develop RIDNs, we asked whether RIDNs formed in Tg-RTN3 mice would occur in a normal, nontransgenic condition of aging. To address this question, brain sections from wild-type (wt) mice that were killed at various ages were prepared for immunostaining with the RTN3-specific antibody, R458. We found that RIDNs in brain sections from mice younger than 6 months of age were not detectable (Fig. $1 A$, panel $6 \mathrm{mth}$ ), and a few sporadic RIDNs were detectable in mice at the age of 12 months (data not shown). Remarkably, the number of RIDNs was clearly increased at the age of 15 months (Fig. $1 \mathrm{~A}$, panel $15 \mathrm{mth})$. At the age of 24 months, RIDNs were abundantly present in every examined sample (Fig. $1 \mathrm{~A}$, panel $24 \mathrm{mth}$ ). Similar to our observations in young Tg-RTN3 mice, the appearance of RIDNs was mainly restricted to the hippocampal CA1 region, and was much less abundant in the CA3 and dentate gyrus regions (Fig. 

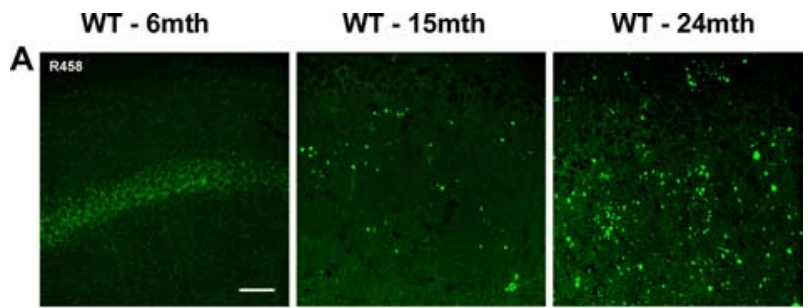

DG

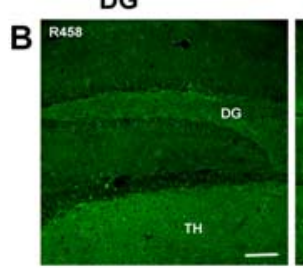

CA3

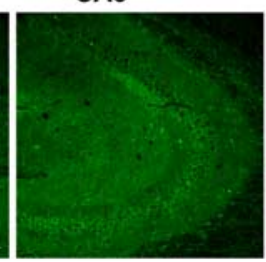

Frontal Cortex

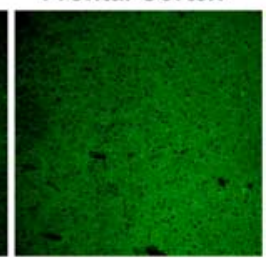

C

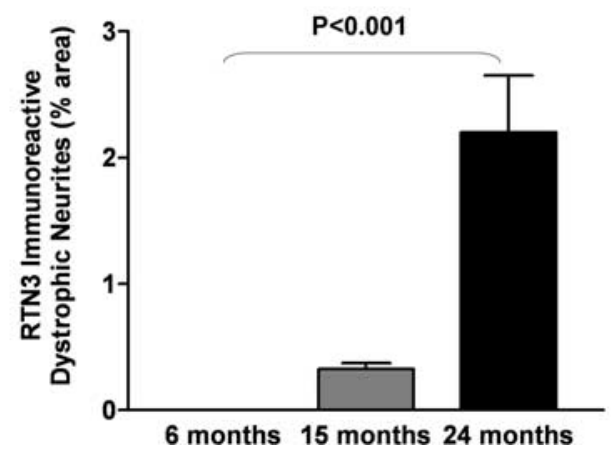

Figure 1. RTN3 labels dystrophic neurites in elderly mouse brain. $A$, Sections of brain tissues from three age groups (6, 15, and 24 months old) were immunostained with antibody R458, which recognizes the $C$ termini of RTN3. The age-dependent occurrence of RIDNs was observed in the $C A 1$ region. $B, 0$ ther brain regions from 24-month-old mice sections showed either significantly less RIDNs or no RIDNs. Scale bars: $\boldsymbol{A}, 30 \mu \mathrm{m} ; \boldsymbol{B}, 75 \mu \mathrm{m}$. $\boldsymbol{C}$, The percentage of area of the hippocampal CA1 region containing RIDNs from different ages of mice was calculated using ImageJ software. Eight sections randomly selected from each animal were stained and quantified. $n=3$ animals, $p<0.001$, Student's $t$ test.

$1 B$; 24-month-old). Further quantification of RIDNs in the CA1 region showed a consistent and significant increase of RIDNs during the aging process (Fig. $1 C)(n=3$ animals per group, 8 sections per animal).

To examine whether RIDNs in aged mouse brains would contain other reported dystrophic neurite markers, we performed double-label staining using brain sections from 24-month-old mice. Antibody SMI31, previously shown to mark dystrophic neurites in the elderly human brain (Vickers et al., 1996), was first chosen for the costaining. Noticeably, SMI31 not only reacted normally with phosphorylated neurofilament $\mathrm{H}$ in cell bodies as well as in thick and thin axons, but also labeled clustered dystrophic neurites (Fig. 2A). As shown clearly in the merged panel, antibody SMI31 did not label dispersed dystrophic neurites, which could only be distinctly marked by the RTN3 antibody in green, whereas the clustered dystrophic neurites labeled by the SMI31 antibody also contained RTN3. Another commonly used antibody to mark dystrophic neurites is the ubiquitin antibody. We found that the ubiquitin antibody also similarly labeled clustered dystrophic neurites (Fig. 2B). As shown in an enlarged view of merged panel, ubiquitin immunoreactivity was apparently smaller in size, and some of the dystrophic neurites were partially
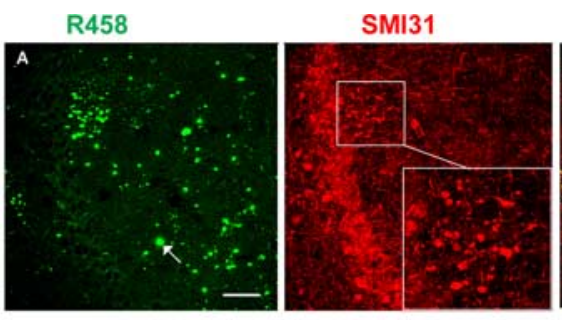

R458/SMI31
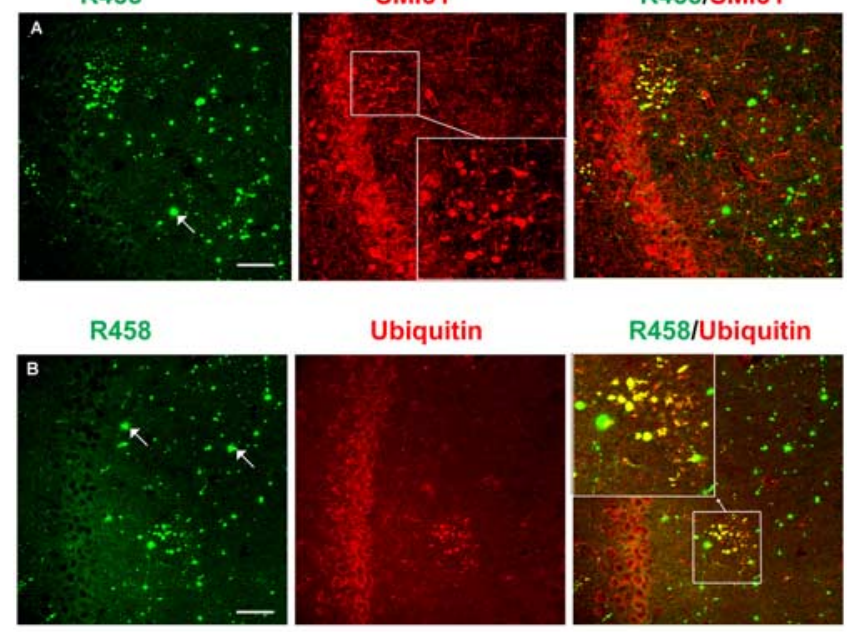

R458/Ubiquitin

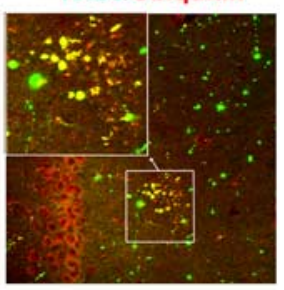

APP

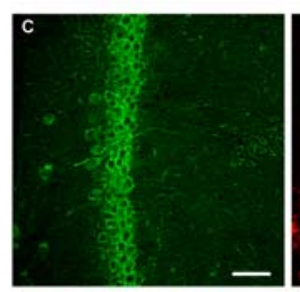

R458

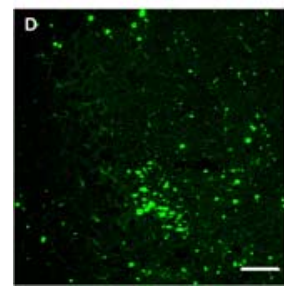

Ubiquitin

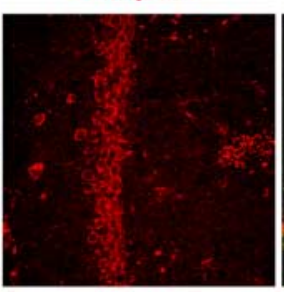

MAP2

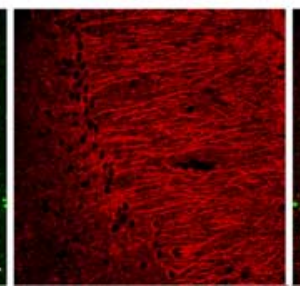

APP/Ubiquitin

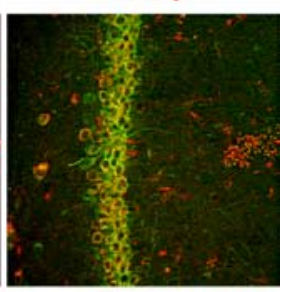

R458/MAP2

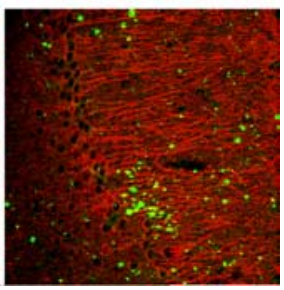

Figure 2. RIDNs represent more abundant dystrophic neurites in the elderly mouse brain. $A-D$, Brain sections from 24-month-old mice were double-labeled with R458 for RTN3 or APP (green) and each of the following antibodies in red: phosphorylated neurofilaments $\mathrm{H}$ (SMI31; $\boldsymbol{A})$, ubiquitin $(\boldsymbol{B}, \boldsymbol{C})$, and dendritic protein MAP2 (D). The red immunoreactivity of SMI31 $(\boldsymbol{A})$ and ubiquitin $(\boldsymbol{B})$ marked only the clustered dystrophic neurites that are also highlighted in the inset. APP is also only enriched in the clustered dystrophic neurites ( $($ ). The green RTN3 immunoreactivity marked all dystrophic neurites, including the large ones specified with arrows. Scale bar, $30 \mu \mathrm{m}$.

buried within the RTN3 immunoreactive signal. In both cases, RIDNs represented a broader population of dystrophic neurites.

APP is a well known axonally transported protein, and antibodies recognizing APP are often used to detect dystrophic neurites in surrounding amyloid plaques in $\mathrm{AD}$ brains (Shoji et al., 1990). A previous study suggested that APP-marked dystrophic neurites could occur before the ubiquitin-marked dystrophic neurites in human aged brain (Cras et al., 1991). Interestingly, we found that an antibody recognizing the APP C terminus still marked only clustered dystrophic neurites, and the APP immunoreactivity appeared in an even smaller size than the overlapping ubiquitin immunoreactivity (Fig. $2 C$ ). It was noted that the antibody recognizing MAP2, which shows selective labeling of dendritic trees, failed to mark dystrophic neurites (Fig. 2D). Another antibody, SMI32, which reacts with nonphosphorylated neurofilament $\mathrm{H}$ predominantly in the cell bodies and dendrites, also failed to mark dystrophic neurites (supplemental Fig. $1 \mathrm{~A}$, 
A

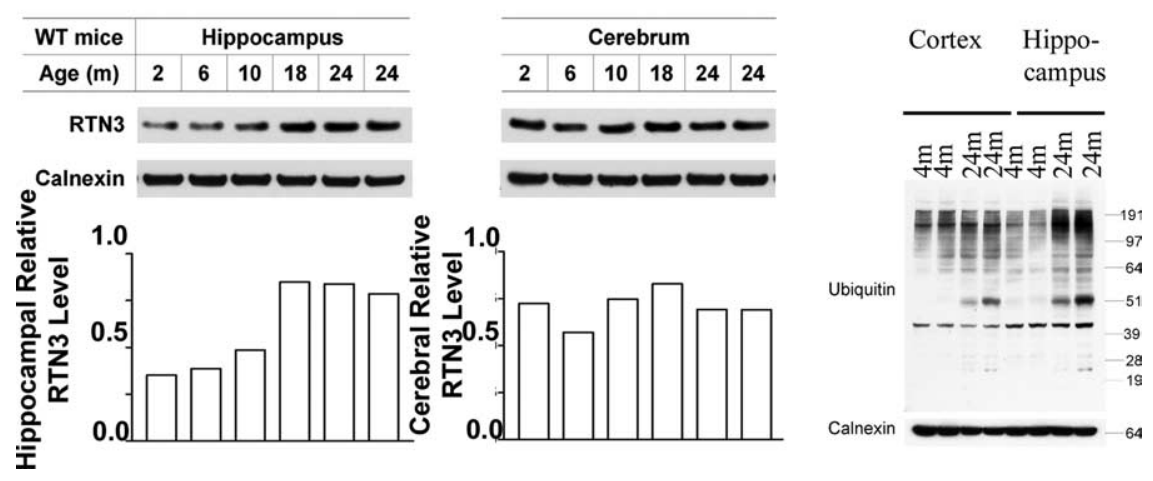

Figure 3. RTN3 protein levels are elevated in elderly mouse brains. $\boldsymbol{A}$, Western analysis of brain tissues from mice at different ages showed increased RTN3 protein levels in 18- and 24-month-old samples. The relative protein levels of RTN3 over calnexin were plotted. $\boldsymbol{B}$, A reduction in the ubiquitin-proteasomal system was evidenced by the enhanced ubiquitinylated protein signals in samples from 24-month-old compared with 4-month-old animals. An ubiquitinylated protein that migrated near $51 \mathrm{kDa}$ was only seen in the 24-month-old mouse samples, and not in the 4-month-old samples.

available at www.jneurosci.org as supplemental material). Hence, these dendritic cytoskeletal proteins were not contained in either dispersed or clustered RIDNs. Despite that the growth cone marker GAP43 and phosphorylated tau, recognized by antibody AT8, were previously shown to mark dystrophic neurites in $\mathrm{AD}$ brains, neither was found to be trapped in dystrophic neurites in the elderly brain in this study (supplemental Fig. $1 B, C$, available at www.jneurosci.org as supplemental material). Dystrophic neurites marked by phosphorylated tau antibody are commonly found in association with neurofibrillary tangles, primarily present in the brains of $\mathrm{AD}$ and tauopathy cases, and rarely found in normal brains.

It appears that, in the aging hippocampus, at least two distinct populations of dystrophic neurites are formed, both of which contain RTN3. Although the reason for the formation of clustered dystrophic neurites is not yet known, they seem enriched with multiple proteins such as RTN3, APP, phosphorylated neurofilament $\mathrm{H}$, and accumulated ubiquitinylated proteins. Altered phosphorylation of neurofilament $\mathrm{H}$ and elevated ubiquitinylation suggest a potential damage of axons (Martin et al., 1990). It should be emphasized that the appearance of the clustered dystrophic neurites is found only in 24-month-old samples, but not in the 15-month-old samples. Whether the occurrence of the clustered dystrophic neurites is a consequence of the buildup of dispersed RIDNs remains to be investigated.

\section{Elevated levels of RTN3 in the elderly brain}

Dispersed, but not clustered, RIDNs are predominantly formed in Tg-RTN3 mice, and their formation is correlated with elevated RTN3 protein levels (Hu et al., 2007). To determine whether protein levels of RTN3 are altered during the aging process, we performed Western blot analysis using brain samples from either cortical or hippocampal tissues. RTN3 protein levels appeared similar between the ages of 2 and 10 months, but were elevated in 18-month-old samples and beyond (Fig. $3 A)(n=3$ independent experiments). This increase was more evident in the hippocampus than in the cerebral cortex. We also found that the level of RTN3 mRNA was slightly elevated during aging (data not shown). A more remarkable alteration was the impaired ubiquitin-proteasome proteolytic system in the elderly brain, in which total ubiquitinylated proteins were increasingly accumulated (Fig. 3B). The impaired ubiquitin-proteasome proteolytic system in elderly mouse brains could contribute to the elevated RTN3 protein levels, as discussed in our previous study ( $\mathrm{Hu}$ et al., 2007).

\section{The accelerated formation of RIDNs in transgenic mouse brain}

As reported previously, Tg-RTN3 mice develop RIDNs in their hippocampal regions (Hu et al., 2007). The demonstration of RIDNs in aged mouse brain suggests that increased expression of RTN3 in transgenic mouse neurons has essentially mimicked the formation of dispersed RIDNs. Since it was unclear how early RIDNs would appear in Tg-RTN3 mice, we examined the age-dependent appearance of RIDNs. Although the RTN3 transgene was expressed even at the embryonic stage in Tg-RTN3 mice (line 3; data not shown), RIDNs were rarely detectable in Tg-RTN3 mice at an age younger than postnatal day 60 (P60) (Fig. 4A). Only a few sporadic RIDNs were found in Tg-RTN3 mice at the age of P75 (data not shown), but an abundant level of RIDNs appeared in the CA1 regions of the transgenic mice at the age of P90 (Fig. 4B) and significantly more at P120 (Fig. 4C). From our comparison, the amount of RIDNs formed in wt mice at the age of 24 months was more than that in P90 Tg-RTN3 mice, but less than that in P120 Tg-RTN3 mice (compare Figs. 1C, 4D), indicating that the formation of RIDNs was markedly accelerated in the case of the Tg-RTN3 mice. This acceleration could apparently be attributable to both an age-dependent factor and the increased expression of the RTN3 transgene in Tg-RTN3 mice, in which $\sim 4$-fold more RTN3 was expressed (Hu et al., 2007).

\section{Reduction in dendritic spines following formation of RIDNs}

The presence of abundant RIDNs in the CA1 region of Tg-RTN3 mice causes a reduction in long term potentiation (LTP) (Hu et al., 2007). This reduction suggests that an EPSP is possibly impaired. Since hippocampal excitatory synapses are directly correlated with dendritic spine density (Harris and Kater, 1994; Moser et al., 1994), we hypothesized that the occurrence of RIDNs would lead to a loss of afferent synaptic terminal signals and further reduce dendritic spine density. To test this hypothesis, we performed Golgi-staining on brain samples from two different age groups of mice: 2 months old and 10 months old. The dendritic arborization and spine density were not obviously different before the formation of RIDNs in 2-month-old Tg-RTN3 mice compared with their wt littermates (data not shown). However, dendritic spine structures in Tg-RTN3 mice at the age of 10 months were clearly altered compared with wt mice (Fig. $5 A-H$, compare wt in the left panels to Tg-RTN3 in the right panels). Specifically, the basal and apical dendritic arborization in the transgenic mice was significantly less extended, and dendritic segments in both apical and basal trees were dramatically fewer in the Tg-RTN3 CA1 region. In contrast, this difference was less obvious in the CA3 region. Closer examination of dendritic spine densities of both basal and apical segments showed that there were visibly fewer spines in Tg-RTN3 mice than in wtcomparable segments (Fig. 5G,H). This typical reduction was not seen in Tg-RTN3 mice that had not yet developed RIDNs (Fig. $5 I)$. Moreover, a dendritic spine loss of $\sim 50 \%(20.47 \pm 0.9$ in wt vs $10.27 \pm 0.61$ in Tg-RTN3 CA1; $n=15, p<0.001$ ) was only 

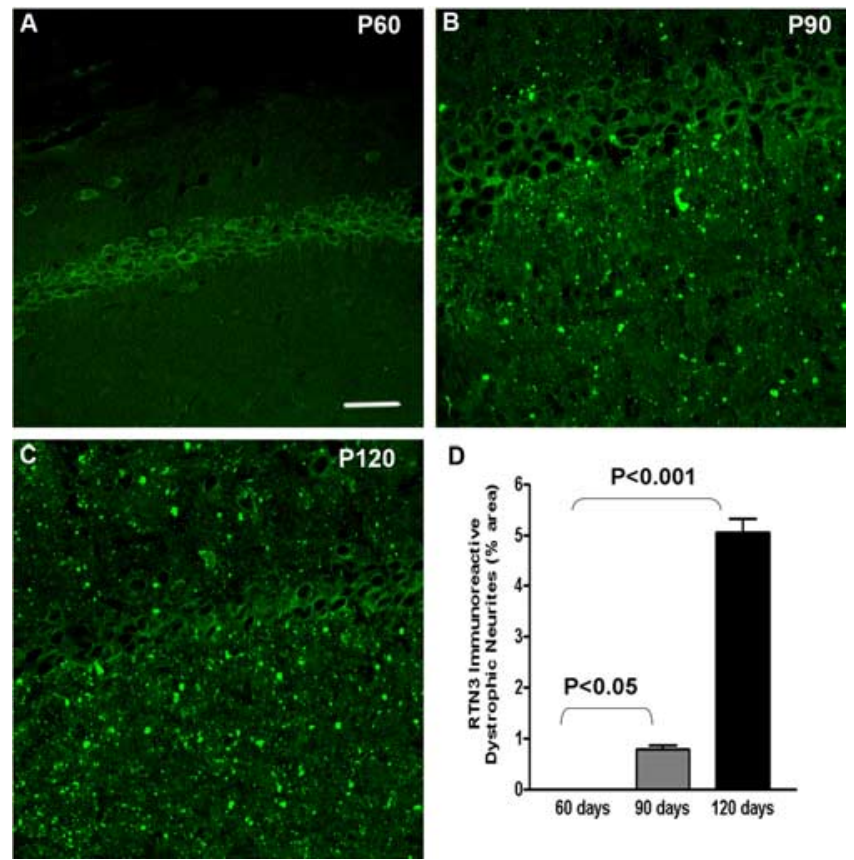

D

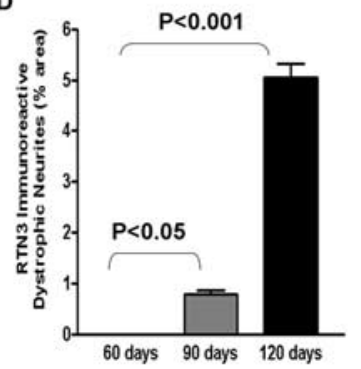

Figure 4. Age-dependent development of RIDNs in Tg-RTN3 mice. Brain sections from TgRTN3 mice were immunostained with RTN3 antibody R458. Abundant RIDNs were found in mice older than P90, but not at an earlier age. Scale bar, $30 \mu \mathrm{m}$. B, The percentage of area of RIDNs occupied in the Tg-RTN3 hippocampal CA1 region was calculated using ImageJ software. Eight sections randomly selected from each animal were stained and quantified. $n=3$ animals. $p<$ 0.05 at $90 \mathrm{~d}$ and $p<0.01$ at $120 \mathrm{~d}$.

seen in CA1 pyramidal neurons, whereas the reduction was not significant in the $\mathrm{CA} 3$ region $(18.63 \pm 1.06$ in wt vs $17.88 \pm 1.34$ in Tg-RTN3 CA1; $n=8, p=0.668$ ). Our results therefore indicate that the occurrence of RIDNs causes the loss of dendritic spines, a phenomenon that indeed exists in the elderly brain (Duan et al., 2003; Dickstein et al., 2007).

\section{Increased levels of RTN3 impair anterograde} axonal transport

To explore the mechanism underlying the increased levels of RTN3 in forming aggregates and dystrophic neurites, we tested whether an unbalanced axonal transport might create an abnormal environment that compels RTN3 to form aggregates. We reasoned that RTN3 is likely a cargo protein involved in the axonal transport of vesicles, resulting from the localization of RTN3 in axons and growth cones (Hu et al., 2007). If this is the case, increased expression of RTN3 could perhaps lead to excessive axonal transport and cause an impairment of the axonal transport machinery.

Ligation of sciatic nerves is an established approach used for the study of transportation of molecules in mammalian axons. We examined ligated mouse sciatic nerves and compared levels of RTN3 in the segments immediately proximal to the ligature with those immediately distal to it, and found that the level of RTN3 was slightly higher in the proximal than in the distal segments (Fig. 6A). This suggests that RTN3 can normally be anterogradely transported. Compared with proteins known to undergo fastanterograde transport, we found that both APP and synapsin-I were more rapidly accumulated in the proximal segments of the ligated nerve, whereas little remained in the distal end (Fig. 6A). Since accumulation of RTN3 in the proximal ligature was less prominent than the above two proteins, we compared the axonal transport of RTN3 with that of neurotrophin receptor p75, which

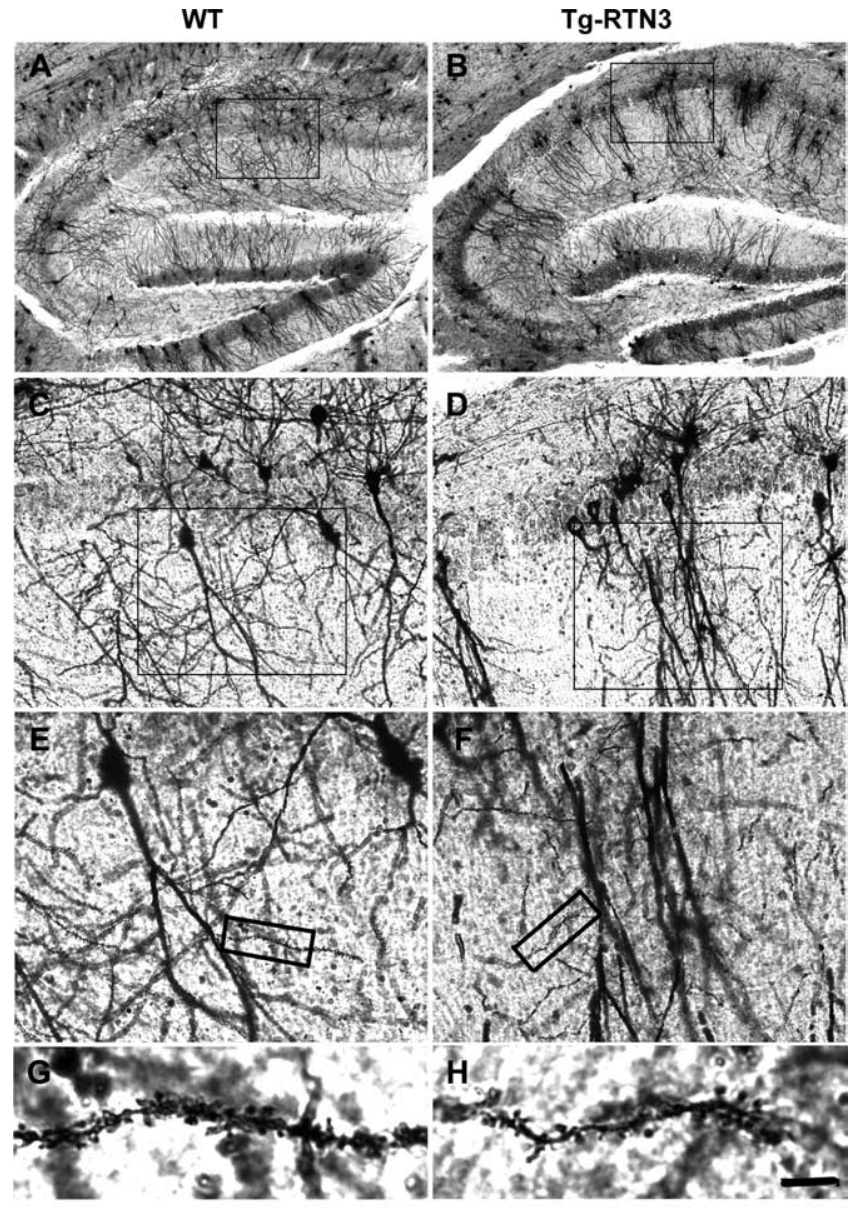

I
2 months

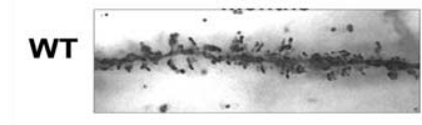

TgRTN3

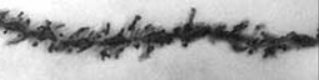

10 months
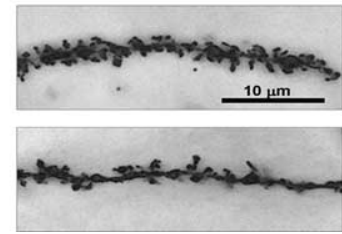

Figure 5. RIDNs impair dendritic arborization and reduce dendritic spine density in hippocampal CA1 region. The Golgi stain was used to label pyramidal neurons and their dendrites in the brains of 10-month-old mice. $\boldsymbol{A}-\boldsymbol{H}$, Representative pictures from a wild-type mouse are shown in $A, C, E$, and $G$, whereas representative pictures from a Tg-RTN3 mouse are shown in $B$, $\boldsymbol{D}, \boldsymbol{F}$, and $\boldsymbol{H}$. Scale bar: (in $\boldsymbol{H}) \boldsymbol{A}, \boldsymbol{B}, 200 \mu \mathrm{m} ; \boldsymbol{C}, \boldsymbol{D}, 50 \mu \mathrm{m} ; \boldsymbol{E}, \boldsymbol{F}, 25 ; \boldsymbol{G}, \boldsymbol{H}, 5 \mu \mathrm{m}$. Areas identified by boxes in images are shown at higher magnification in the images below them. Compared with the wild-type mouse $(\boldsymbol{A})$, the dendritic trees of pyramidal neurons in the CA1 region of the Tg-RTN3 mouse often spread unevenly and formed irregular bundles $(\boldsymbol{B})$. They had much less secondary and tertiary dendritic branches, resulting in loss of dendritic networks $(\boldsymbol{B})$. In contrast, the difference was not obvious in the CA3 region between the two groups of mice. The pictures of dendritic arborization of pyramidal neurons were enlarged $(\boldsymbol{C}-\boldsymbol{F})$. The dendritic branches of the wild type extended smoothly and widely $(\boldsymbol{C}, \boldsymbol{E})$, whereas those of the Tg-RTN3 were unsmooth and scarce, indicating dendritic impairment in the Tg-RTN3 mice $(\boldsymbol{D}, \boldsymbol{F})$. The density of dendritic spines was clearly reduced in the $\operatorname{Tg}$-RTN3 mice $(\boldsymbol{H})$ compared with the wild type $(\boldsymbol{G}) . \boldsymbol{I}$, Representative segments of Golgi-stained dendritic tertiary branches were all taken from the basal dendrites. The density of dendritic spines was clearly reduced in 10-month-old Tg-RTN3 mice compared with the wt control. Such a reduction was actually present in both apical and basal dendrites. The number of dendritic spines was quantified from randomly selected dendritic segments of $10 \mu \mathrm{m}$ length at the age of 10 months, and a reduction of $\sim 50 \%$ was found in the CA1 region ( $p<0.0001$, unpaired $t$ test, $n=15$ ), but not the CA3 region ( $p>0.668$, unpaired $t$ test, $n=8$ ) of Tg-RTN3 mice compared with the wt controls. 


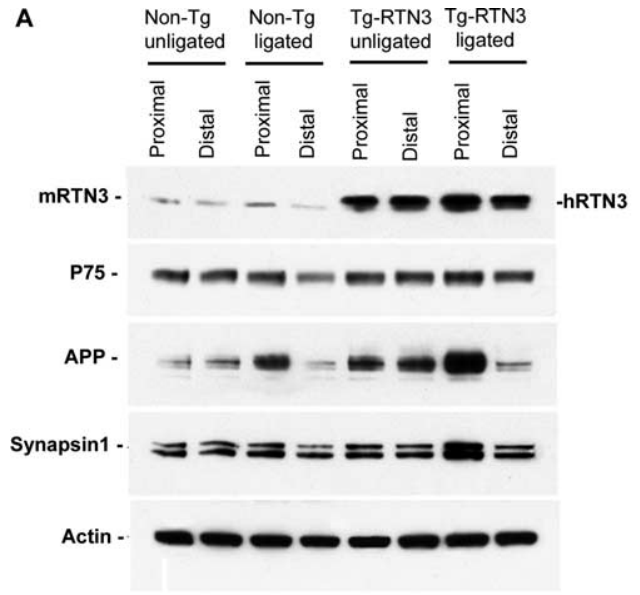

B

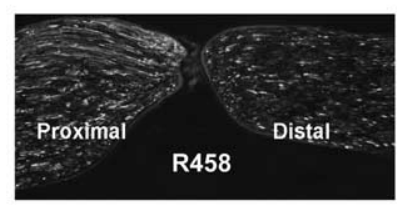

C
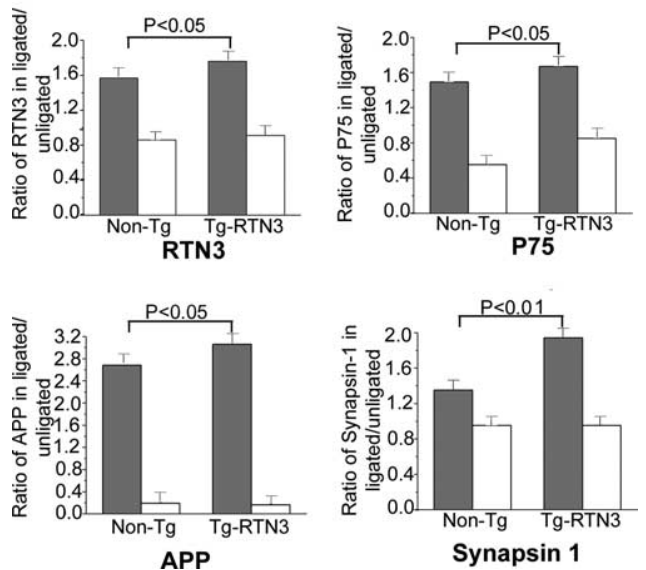

Figure 6. Impaired axonal transport in Tg-RTN3 axons. A, Proteins transported along axons in 8-month-old wt mice and Tg-RTN3 mice were examined. The sciatic nerves were first subjected to double ligatures to ensure complete blockage of axonal transport. Equal amounts of protein extracts from the indicated segment were examined by Western analysis using antibody R458 to detect RTN3. The same blot was reacted with antibodies against neurotrophin receptor p75, APP and synapsin-I. Anti-actin was used to show equal loading of samples. $\boldsymbol{B}$, Cryostat sections of the proximal and distal ends of ligated sciatic nerves of Tg-RTN3 line 3 mice were labeled with antibody R458 to show the distribution of RTN3 within the ligated segments. The nerve ligation was allowed to proceed for $20 \mathrm{~h}$. C, The ratio of the specified protein in ligated over unligated proximal (filled bar) or distal (open bar) segment was quantified based on the analysis of RTN3 protein levels on the Western blot. The results are representative of three pairs of animals. $p<0.05$ for RTN3, p75, and APP and $p<0.01$ for synapsin-I, Student's $t$ test.

was previously shown to be transported both anterogradely and retrogradely (Yano and Chao, 2004). The accumulation of p75 at the proximal ligature was clearly similar to that of RTN3, and significantly less than that of APP and synapsin-I (Fig. 6A). Thus, our data suggests that RTN3 is not only an anterogradely transported protein, but also a retrogradely transported protein. This suggests that normally the transport of RTN3 is a delicate equilibrium in both directions.

The anterograde transport of RTN3 became obvious after TgRTN3 mouse sciatic nerves were ligated for $6 \mathrm{~h}$ (Fig. 6A), and more evident if the nerve was ligated for $24 \mathrm{~h}$ as shown by confocal examination of the ligated segments detected by antibody
R458 (Fig. 6B). This observation suggests that increased expression of RTN3 leads to accelerated transport via the anterograde pathway. Comparing the ratio of RTN3 levels in the ligated versus the unligated immediately proximal segments indicated that there was visibly more RTN3 accumulated in Tg-RTN3 mice than in the nontransgenic control mice (Fig. $6 C)(n=3)$. Accumulation of APP and synapsin-I in the proximal segments of Tg-RTN3 mouse nerves was also markedly increased compared with the nontransgenic controls (Fig. 6A), suggesting that increased expression of RTN3 either produces greater transport of APP and synapsin-I in axons, or enhances the velocity of anterograde transport. Given these findings, we believe that the specific axonal transport of RTN3 is altered because of an imbalance in the anterograde and retrograde transportation in Tg-RTN3 mice, and that this imbalance may potentially cause RTN3 to accumulate in the axonal terminus, in which RTN3 has a tendency to form aggregates and to form RIDNs.

\section{Discussion}

Aging-dependent functional decline, including reduced memory, is commonly encountered by normal elderly people (Winocur and Moscovitch, 1990; Gallagher and Rapp, 1997). Various studies have linked age-dependent cognitive decline to deficits in synaptic transmission and plasticity (deToledo-Morrell et al., 1988; Campbell et al., 1996; Smith et al., 2000). These deficits are not caused by neuronal loss or volumetric changes in the elderly brain (Rapp et al., 1999; Morrison and Hof, 2007), nor are they attributable to any single neurological factor, according to the gene expression profiling results (Blalock et al., 2003; Verbitsky et al., 2004). In this study, we have discovered that abundant RIDNs are specifically formed in the hippocampal CA1 region of elderly mouse brains, a critical region for learning and memory. The occurrence of RIDNs indeed causes impaired hippocampal function as further evidenced by the loss of dendritic spines (Fig. 5). Hence, pharmacological inhibition of the formation of RIDNs may be an effective strategy for ameliorating cognitive decline in the elderly.

As demonstrated, abundant dystrophic neurites are present in a dispersed form and can only be marked by RTN3 antibody, and this form of RIDNs develops in young Tg-RTN3 mice. Biochemically, the dispersed RIDNs contain RTN3 aggregates (Hu et al., 2007). The abundance of RIDNs in the hippocampus of 24month-old wt mice is comparable to that seen in Tg-RTN3 mice between the ages of 3 and 4 months, and RTN3 protein levels are indeed increased in 24-month-old mouse brain. Hence, spontaneous development of RIDNs in Tg-RTN3 and elderly mouse brains is directly dependent on higher RTN3 levels. This strongly advocates for the Tg-RTN3 mouse as a useful model to study the age-dependent formation of RIDNs.

Occurrence of dispersed neuritic dystrophy marked by synucleins was observed in the hippocampus of Parkinson's disease and Lewy body dementia (Galvin et al., 1999). However, the accumulation of synucleins in dystrophic neurites appears more restricted to the above diseases, especially in the case of $\beta$ - and $\gamma$-synucleins. Dystrophic neurites occurring in AD cases are found to trap many proteins, including ubiquitin, APP and neurofilament proteins, but the accumulation of these proteins is mainly caused by the deposition of amyloid plaques (Lenders et al., 1989; Shoji et al., 1990; Cras et al., 1991). In this study, we found that the population of dystrophic neurites marked by ubiquitin, SMI31, and APP is rarely detected in mouse brain at the age of 15 months, but appeared in a clustered form in much older mice (24-month-old), in line with previous observations 
(Migheli et al., 1992; Vickers et al., 1996). Their occurrence is likely resulting from an axonal alteration because they are not marked by SMI32, which recognizes nonphosphorylated neurofilament $\mathrm{H}$ in dendrites, and they are also devoid of another dendritic marker, MAP2. Within the clustered neurites, connection of individual swollen puncta is visible (Fig. 2A), suggesting that clustered dystrophic neurites are likely to originate from bifurcated axonal branches. Potential structural alterations in these local areas may trigger dramatic biochemical changes, including accumulation of phosphorylated neurofilament $\mathrm{H}$ and ubiquitinylated proteins that are accumulated in these neurites. RTN3 can also clearly be accumulated in these clustered neurites, but whether overexpression of RTN3 will trigger this damage remains to be investigated.

Our mechanistic study suggests that higher RTN3 levels cause impairment in axonal transport. Perhaps, RTN3 is a structural membrane protein of a vesicle that can be transported via kinesin-1-mediated machinery, as shown in Figure 6. When the levels of RTN3 are high, the quantity of RTN3-containing vesicles is perhaps increased or more RTN3 is incorporated into these RTN3-containing vesicles. Therefore, the transport velocity of these vesicles is likely more dependent on the concentration of RTN3 in the anterograde direction, but less so in the retrograde direction. This may result in an imbalance in the anterograde and retrograde transport systems. In this study, we showed that significantly more APP and synapsin-I were accumulated in the proximal ligature when sciatic nerves of Tg-RTN3 mice were ligated for only $6 \mathrm{~h}$, compared with the nontransgenic control (Fig. 6). It should be noted that the total protein levels of synapsin-I and p75 in unligated nerves remained essentially the same in the two genotype groups, but the level of APP was evidently increased in Tg-RTN3 mice compared with the controls (Fig. 6A). The increased level of APP was consistent with the original in vitro finding of RTN3 as a BACE1 negative modulator (He et al., 2004, 2006, 2007; Murayama et al., 2006; Wojcik et al., 2007). APP is suggested to be a cargo protein of an axonal transport machinery (Goldstein, 2003), and its elevated level may contribute to the accelerated axonal transport of proteins including APP, and perhaps also synapsin-I. Nevertheless, the subsequent accumulation of RTN3 in the neuritic regions may increase the opportunity for RTN3 to form high molecular weight (HMW)RTN3 that is deposited in the neuritic regions. Although additional experiments are required to fully test the above hypothesis, the findings of membrane stacks near RTN3-containing filamentous aggregates and in vitro evidence of RTN3 in forming aggregates (Hu et al., 2007) partially support the above model.

As demonstrated in this study, the occurrence of dystrophic neurites can be formed before detectable axonal or neuronal injuries or other neurodegenerative features. Appearance of such abundant RIDNs should raise concerns for hippocampal function. Indeed, we have shown that the appearance of RIDNs in our mouse model causes reduced spine density and altered dendritic organization. Reduced spine density may stem from reduced presynaptic signaling inputs caused by the accumulation of RTN3 aggregates in the swollen axonal terminals. Reduced spine density has been shown to correlate with the loss of afferent synaptic terminals as well as reduced LTP (Moser et al., 1994; Muller et al., 2000; Connor et al., 2006). In the elderly brain, loss of spine density has been reported (Duan et al., 2003; Dickstein et al., 2007), and an abundance of RIDNs in the elderly brain could contribute to reduced synaptic function and cognitive decline.

Aging is a major risk factor for $\mathrm{AD}$ pathogenesis. Agedependent occurrence of abundant RIDNs could certainly con- tribute to the cognitive dysfunction in both normal elderly people as well as in AD patients. Although it is not understood how dispersed RIDNs become clustered around neuritic plaques, a higher density of RIDNs compared with amyloid plaques was recently found in the hippocampal CA1 region of the brain from an AD case (supplemental Fig. 2, available at www.jneurosci.org as supplemental material). More importantly, we have recently shown in our animal studies that preformed RIDNs will adversely affect amyloid deposition (Shi Q, Prior M, He W, Hu X, Yan R, unpublished observations). Hence, the abundant RIDNs formed before amyloid deposition in human hippocampus may, in turn, potentially contribute to increased amyloid deposition and reduced cognitive function. Inhibition of the formation of RIDNs would therefore have a therapeutic and preventive advantage in the treatment of AD.

\section{References}

Blalock EM, Chen KC, Sharrow K, Herman JP, Porter NM, Foster TC, Landfield PW (2003) Gene microarrays in hippocampal aging: statistical profiling identifies novel processes correlated with cognitive impairment. J Neurosci 23:3807-3819.

Campbell LW, Hao SY, Thibault O, Blalock EM, Landfield PW (1996) Aging changes in voltage-gated calcium currents in hippocampal CA1 neurons. J Neurosci 16:6286-6295.

Connor S, Williams PT, Armstrong B, Petit TL, Ivanco TL, Weeks AC (2006) Long-term potentiation is associated with changes in synaptic ultrastructure in the rat neocortex. Synapse 59:378-382.

Cras P, Kawai M, Lowery D, Gonzalez-DeWhitt P, Greenberg B, Perry G (1991) Senile plaque neurites in Alzheimer disease accumulate amyloid precursor protein. Proc Natl Acad Sci U S A 88:7552-7556.

deToledo-Morrell L, Geinisman Y, Morrell F (1988) Age-dependent alterations in hippocampal synaptic plasticity: relation to memory disorders. Neurobiol Aging 9:581-590.

Dickson DW, Farlo J, Davies P, Crystal H, Fuld P, Yen SH (1988) Alzheimer's disease. A double-labeling immunohistochemical study of senile plaques. Am J Pathol 132:86-101.

Dickson DW, Wertkin A, Kress Y, Ksiezak-Reding H, Yen SH (1990) Ubiquitin immunoreactive structures in normal human brains. Distribution and developmental aspects. Lab Invest 63:87-99.

Dickson TC, King CE, McCormack GH, Vickers JC (1999) Neurochemical diversity of dystrophic neurites in the early and late stages of Alzheimer's disease. Exp Neurol 156:100-110.

Dickstein DL, Kabaso D, Rocher AB, Luebke JI, Wearne SL, Hof PR (2007) Changes in the structural complexity of the aged brain. Aging Cell 6:275-284

Duan H, Wearne SL, Rocher AB, Macedo A, Morrison JH, Hof PR (2003) Age-related dendritic and spine changes in corticocortically projecting neurons in macaque monkeys. Cereb Cortex 13:950-961.

Gallagher M, Rapp PR (1997) The use of animal models to study the effects of aging on cognition. Annu Rev Psychol 48:339-370.

Galvin JE, Uryu K, Lee VM, Trojanowski JQ (1999) Axon pathology in Parkinson's disease and Lewy body dementia hippocampus contains alpha-, beta-, and gamma-synuclein. Proc Natl Acad Sci U S A 96:13450-13455.

Goldstein LS (2003) Do disorders of movement cause movement disorders and dementia? Neuron 40:415-425.

Harris KM, Kater SB (1994) Dendritic spines: cellular specializations imparting both stability and flexibility to synaptic function. Annu Rev Neurosci 17:341-371.

He W, Lu Y, Qahwash I, Hu XY, Chang A, Yan R (2004) Reticulon family members modulate BACE1 activity and amyloid-beta peptide generation. Nat Med 10:959-965.

He W, Hu X, Shi Q, Zhou X, Lu Y, Fisher C, Yan R (2006) Mapping of interaction domains mediating binding between BACE1 and RTN/Nogo Proteins. J Mol Biol 363:625-634.

He W, Shi Q, Hu X, Yan R (2007) The membrane topology of RTN3 and its effect on binding of RTN3 to BACE1. J Biol Chem 282:29144-29151.

Hu X, Shi Q, Zhou X, He W, Yi H, Yin X, Gearing M, Levey A, Yan R (2007) Transgenic mice overexpressing reticulon 3 develop neuritic abnormalities. EMBO J 26:2755-2767.

Irizarry MC, Growdon W, Gomez-Isla T, Newell K, George JM, Clayton DF, 
Hyman BT (1998) Nigral and cortical Lewy bodies and dystrophic nigral neurites in Parkinson's disease and cortical Lewy body disease contain alpha-synuclein immunoreactivity. J Neuropathol Exp Neurol 57:334-337.

Kowall NW, Kosik KS (1987) Axonal disruption and aberrant localization of tau protein characterize the neuropil pathology of Alzheimer's disease. Ann Neurol 22:639-643.

Lenders MB, Peers MC, Tramu G, Delacourte A, Defossez A, Petit H, Mazzuca M (1989) Dystrophic neuropeptidergic neurites in senile plaques of Alzheimer's disease precede formation of paired helical filaments. Acta Neurol Belg 89:279-285.

Liberski PP (1995) Reappraisal of ultrastructural diversity of amyloid plaques in Gerstmann-Straussler-Scheinker syndrome. Pol J Pathol 46:33-41.

Martin JE, Mather KS, Swash M, Garofalo O, Dale GE, Leigh PN, Anderton BH (1990) Spinal cord trauma in man: studies of phosphorylated neurofilament and ubiquitin expression. Brain 113:1553-1562.

Masliah E, Mallory M, Hansen L, Alford M, DeTeresa R, Terry R, Baudier J, Saitoh T (1992) Localization of amyloid precursor protein in GAP43immunoreactive aberrant sprouting neurites in Alzheimer's disease. Brain Res 574:312-316.

Migheli A, Attanasio A, Vigliani MC, Schiffer D (1991) Dystrophic neurites around amyloid plaques of human patients with Gerstmann-StrausslerScheinker disease contain ubiquitinated inclusions. Neurosci Lett 121:55-58.

Migheli A, Attanasio A, Pezzulo T, Gullotta F, Giordana MT, Schiffer D (1992) Age-related ubiquitin deposits in dystrophic neurites: an immunoelectron microscopic study. Neuropathol Appl Neurobiol 18:3-11.

Morrison JH, Hof PR (2007) Life and death of neurons in the aging cerebral cortex. Int Rev Neurobiol 81:41-57.

Moser MB, Trommald M, Andersen P (1994) An increase in dendritic spine density on hippocampal CA1 pyramidal cells following spatial learning in adult rats suggests the formation of new synapses. Proc Natl Acad Sci U S A 91:12673-12675.

Muller D, Toni N, Buchs PA (2000) Spine changes associated with longterm potentiation. Hippocampus 10:596-604.

Murayama KS, Kametani F, Saito S, Kume H, Akiyama H, Araki W (2006) Reticulons RTN3 and RTN4-B/C interact with BACE1 and inhibit its ability to produce amyloid beta-protein. Eur J Neurosci 24:1237-1244.

Oertle T, Schwab ME (2003) Nogo and its paRTNers. Trends Cell Biol 13:187-194

Onorato M, Mulvihill P, Connolly J, Galloway P, Whitehouse P, Perry G
(1989) Alteration of neuritic cytoarchitecture in Alzheimer disease. Prog Clin Biol Res 317:781-789.

Perry G, Friedman R, Shaw G, Chau V (1987) Ubiquitin is detected in neurofibrillary tangles and senile plaque neurites of Alzheimer disease brains. Proc Natl Acad Sci U S A 84:3033-3036.

Praprotnik D, Smith MA, Richey PL, Vinters HV, Perry G (1996) Plasma membrane fragility in dystrophic neurites in senile plaques of Alzheimer's disease: an index of oxidative stress. Acta Neuropathol 91:1-5.

Rapp PR, Stack EC, Gallagher M (1999) Morphometric studies of the aged hippocampus: I. Volumetric analysis in behaviorally characterized rats. J Comp Neurol 403:459-470.

Shoji M, Hirai S, Yamaguchi H, Harigaya Y, Kawarabayashi T (1990) Amyloid beta-protein precursor accumulates in dystrophic neurites of senile plaques in Alzheimer-type dementia. Brain Res 512:164-168.

Smith TD, Adams MM, Gallagher M, Morrison JH, Rapp PR (2000) Circuit-specific alterations in hippocampal synaptophysin immunoreactivity predict spatial learning impairment in aged rats. J Neurosci 20:6587-6593.

Stokin GB, Lillo C, Falzone TL, Brusch RG, Rockenstein E, Mount SL, Raman R, Davies P, Masliah E, Williams DS, Goldstein LS (2005) Axonopathy and transport deficits early in the pathogenesis of Alzheimer's disease. Science 307:1282-1288.

Verbitsky M, Yonan AL, Malleret G, Kandel ER, Gilliam TC, Pavlidis P (2004) Altered hippocampal transcript profile accompanies an agerelated spatial memory deficit in mice. Learn Mem 11:253-260.

Vickers JC, Chin D, Edwards AM, Sampson V, Harper C, Morrison J (1996) Dystrophic neurite formation associated with age-related beta amyloid deposition in the neocortex: clues to the genesis of neurofibrillary pathology. Exp Neurol 141:1-11.

Wang D, Munoz DG (1995) Qualitative and quantitative differences in senile plaque dystrophic neurites of Alzheimer's disease and normal aged brain. J Neuropathol Exp Neurol 54:548-556.

Winocur G, Moscovitch M (1990) Hippocampal and prefrontal cortex contributions to learning and memory: analysis of lesion and aging effects on maze learning in rats. Behav Neurosci 104:544-551.

Wojcik S, Engel WK, Yan R, McFerrin J, Askanas V (2007) NOGO is increased and binds to BACE1 in sporadic inclusion-body myositis and in AbetaPP-overexpressing cultured human muscle fibers. Acta Neuropathol 114:517-526.

Yano H, Chao MV (2004) Mechanisms of neurotrophin receptor vesicular transport. J Neurobiol 58:244-257.

Yan R, Shi Q, Hu X, Zhou X (2006) Reticulon proteins: emerging players in neurodegenerative diseases. Cell Mol Life Sci 63:877-889. 